\title{
A brain network response to sham surgery
}

\author{
Mariya V. Cherkasova and A. Jon StoessI \\ Pacific Parkinson's Research Centre, University of British Columbia, Vancouver, British Columbia, Canada.
}

Evaluation of potential therapies for neurological disease has been challenging due to beneficial responses in patients receiving the sham/placebo treatment. Placebo effects are especially prominent in Parkinson's disease (PD), which has become a useful model for studying the neurobiology of placebo responses. In this issue of the $\mathrm{JCl}$, Ko and colleagues identify a neural circuit associated with the placebo response in a PD patient cohort. The observed placebo effect-associated pattern involved metabolic activity increases that corresponded with long-term motor improvements after sham surgery. Presurgery activity in this network was inversely related to sham response, suggesting that this network has potential for identifying sham responders and thus reducing placebo-related variance in surgical trials.
The placebo effect: a beneficial response to sham treatment

A placebo effect is the physiological and/ or psychological response to an inert intervention that is believed to be therapeutic. Placebo responses emerge in both clinical and research contexts and can assume a variety of forms, including analgesic, antidepressant, and anxiolytic effects, and, in the case of Parkinson's disease (PD), improved motor function. While they may appear to be mysterious, placebo responses have discernible neurophysiological mechanisms.

Placebo responses and their active therapy counterparts share some neurobiological pathways (Table 1); however, treatment and placebo also elicit distinct neural responses. Thus, an active agent and a placebo may modulate components of their shared circuitry to different degrees (1), or each may engage additional regions outside the shared circuitry $(2,3)$. Moreover, different placebo responses appear to have some neural substrates in common. For example, the ventral striatum, which participates in the prediction of rewards - and likely the prediction of therapeutic benefits, has been implicated in placebo analgesia, antidepressant effects (in early treatment phases), and motor improvement in PD. Limbic and paralimbic emotion-processing regions have been associated with both antidepressant and anxiolytic placebo effects, and the rostral anterior cingulate cortex (rACC) is also involved in placebo analgesia. Pathways shared among different placebo responses may reflect their generic launching mechanisms, such as reward anticipation and affect modulation, while pathways shared between placebo and active treatment responses might represent the final effector mechanisms that underlie clinical benefit.

\section{PD: a model for understanding placebo effects}

The central pathology of PD is the degeneration of nigrostriatal dopaminergic projections, which presents clinically as a variety of symptoms, including reduced motor function, tremor, and impaired speech. Placebo effects in PD are prominent following a variety of medical and surgical interventions (4) and have provided a valuable model for studying the underlying neurobiology of placebo-induced

\section{Related Article: p. 3656}

improvement. In patients, the suggestion of anti-Parkinsonian treatment is sufficient to result in striatal dopamine release $(5,6)$ and modulation of abnormal patterns of neuronal firing in the basal ganglia circuitry (7). Furthermore, these neurobiological changes are associated with shortterm clinical improvement.

While the striatal release of dopamine in response to placebo provides an obvious mechanism for both expectation of benefit and short-term motor improvement in PD, there is extensive evidence that placebo responses can be sustained, particularly in surgical trials, though it is not clear how this occurs. In this issue, the study by Ko and colleagues advances our understanding of placebo effects in PD and suggests a brain network associated with longterm clinical benefit from sham surgery (8). The sham surgery was performed on PD patients who were enrolled in a randomized controlled trial (RCT) of a gene therapy designed to normalize firing patterns in the subthalamic nucleus (9). Using fluorodeoxyglucose (FDG) PET to image resting-state brain glucose metabolism, Ko et al. identified a metabolic covariance pattern associated with motor improvements 6 months after sham surgery (8). Sham responders, those individuals who showed significant clinical improvement, displayed activity increases in the limbic and paralimbic regions, including the rostral anterior cingulate, subgenual cingulate, hippocampus, parahippocampal gyrus, and amygdala, as well as the posterior cerebellar vermis. The network also included less reliable contributions from the caudate and thalamus. These network changes were not observed in sham nonresponders, who made up about 30\% of the sham-treated subjects. Of note, the threshold for defining treatment response was quite liberal: an improvement of greater than or equal to 2 points $(5 \%)$ on the Unified Parkinson's Disease Rating Scale (UPDRS) motor subscale. Network expression may have been related to therapeutic expectancy, as network changes were reversed in the majority of respond- 


\section{Table 1. Neurobiological mechanisms of placebo responses}

\begin{tabular}{|c|c|}
\hline Placebo effect & Neurobiological \\
\hline Analgesia & $\begin{array}{l}\text { Activation of endo } \\
\text { Modulation of pai } \\
\text { Striatal dopamine }\end{array}$ \\
\hline Motor improvement in PD & $\begin{array}{l}\text { Striatal dopamine } \\
\text { Modulation of neu }\end{array}$ \\
\hline Antidepressant & $\begin{array}{l}\text { Modulation of acti } \\
\text { Increased activity } \\
\text { Decreased activity }\end{array}$ \\
\hline Anxiolytic & $\begin{array}{l}\text { Activity modulatio } \\
\text { Increased activity } \\
\text { Decreased activity }\end{array}$ \\
\hline Immunosuppression & Conditioning of im \\
\hline Respiratory depression & Conditioning of \\
\hline \multicolumn{2}{|c|}{$\begin{array}{l}\text { ers after they were unblinded to their } \\
\text { treatment } 12 \text { months following the sham } \\
\text { surgery; however, it is unclear whether } \\
\text { reversal of the sham-associated network } \\
\text { was accompanied by declines in motor } \\
\text { function. Network activity at baseline was } \\
\text { inversely related to motor outcomes of } \\
\text { sham intervention in the whole sample. }\end{array}$} \\
\hline
\end{tabular}

\section{Significance of a sham- associated network}

What is the significance of this shamrelated network? The components of this network have previously been implicated in placebo responses, though not in PD. Activity modulation in the limbic and paralimbic regions has been associated with placebo-induced antidepressant effects (2) and modulation of negative emotion $(10,11)$. In addition to antidepressant placebo responses, rACC has been repeatedly implicated in placebo analgesia (12), presumably due to its role in the emotionalzmotivational aspect of pain processing or its more general role in expectancy. Though it has not been directly demonstrated, the rACC is well positioned to participate in placebo-induced dopamine signaling, as it receives prominent dopaminergic projections, participates in reward prediction, and is connected with the ventral striatal and limbic regions $(13,14)$. The posterior cerebellar vermis is also involved in emotional processing as part of the cerebellar-limbic circuitry (15), and cerebellar activations have been demonstrated in prior studies of placebo analgesia $(16,17)$. Given the functional- ities of its components, the cerebello-limbic sham network could modulate emotional processes based on therapeutic expectancy. In fact, changes in network expression after sham surgery were negatively related to concurrent depression scores, though this relationship may be mediated by motor improvement, and antidepressant effects are typically associated with activity decreases in the subgenual cingulate (18).

It may be tempting to interpret the sham network as the underlying mediator of expectation of therapeutic benefit, especially given the effects of unblinding. However, the sham response was associated with low network activity at baseline, though baseline scans may have been performed prior to expectation of participation in the trial. For a network that represents a trait-like proneness to positive expectancy, one might hypothesize that this network is more active at baseline in responders, especially in regions related to reward anticipation, such as the anterior cingulate. In addition, active therapy responders did not display any increase in sham network activity during the blinded phase of the study, even though the therapy-associated response should represent a combination (summation or interaction) of verum and placebo responses. While the difference was not statistically significant, network activity actually tended to increase in active therapy responders after they were unblinded to the treatment. Taken together, these observations suggest that sham network activity may represent something other than expectancy.

The data provided by Ko and colleagues (8) do not offer much insight into how sham network activity translates into improved motor function; therefore, the final effector pathway remains obscure. It is doubtful that striatal and thalamic contributions led to motor improvement, as these were small, less reliable, and did not correlate with motor outcomes as isolated loci. The cerebellar node has not been implicated in somatomotor function (15), though it would be of interest to know which aspects of motor function improved following surgery. For example, improvements in tremor or dyskinesias might more likely reflect activity in cerebellar-basal ganglia circuits than improvements in bradykinesia and rigidity (19). A 6-month follow-up may not have been optimal for detecting all the key sham-related metabolic changes. In the RCT, motor improvements in both the active and sham treatment groups were evident at 1 month after surgery, with no subsequent change (9). Long-term placebo responses may have nonuniform time courses, with different circuits engaged during different phases. Thus, at the 6-month time point, sham network expression may reflect the maintenance of a placebo response that was established earlier.

Theoretically, a candidate effector network that could underpin both sham and active therapy responses and that can display modulation observable by FDG PET several months after surgical 
treatments (20) is the PD-related metabolic pattern (PDRP). This network, discovered and validated by the same group of researchers $(21,22)$, is highly characteristic of PD. Clinically effective medical and surgical treatments reduce expression of the PDRP, whereas disease progression is associated with increased network expression (22). Notably, the RCT evaluated by Ko and colleagues was preceded by a smaller phase I open trial of the gene therapy, which reported significant improvements in motor function (23) and concomitant downmodulation of the PDRP (20). Yet, Ko et al. did not report any discernible modulation of PDRP in response to sham therapy (8).

The apparent lack of an association between the sham response and PDRP and the lack of a clear relationship between the sham network and treatment expectancy may relate to how these networks are identified. Ko et al. deliberately restricted the search for sham-related networks to the component of scan variance orthogonal to the PDRP in an effort to control for the effects of disease progression (8). Though Ko and colleagues report a significant increase in PDRP expression in sham subjects in the 6 months following surgery (PDRP $z$ score of $\approx 2.75$ to $\approx 3$ ), this approach essentially allocates the sham network to some residual variance in glucose metabolism. It is worth noting that the sham network accounted for less than $5 \%$ of the variance reported by Ko et al. (8), whereas the PDRP contributes to more than $20 \%$ of subject $x$ voxel variance according to prior work (21), although PDRPrelated variance was not reported in the current paper. The low baseline sham network activity in the sham responders could reflect exclusion of some expectancy-related variance by this orthogonal approach. Moreover, though PDRP expression did not differ as a function of the sham response, it is not clear whether it was related to motor outcomes in the active therapy patients or in the sample as a whole. If so, the orthogonal approach would eliminate important variance.

\section{Conclusions}

If the sham-related network is indeed a reliable correlate of surgical sham response, this could have important implications for clinical trial design. Sur- gical sham effects are substantial, which makes detecting active treatment effects more challenging and necessitates larger patient cohorts. Using Monte Carlo simulations, Ko et al. (8) demonstrated that exclusion of sham-responsive participants based on their baseline sham network activity reduced the required sample size for the gene therapy trial by more than 56\%; however, this approach may be overly simplistic. First, while placebo responsiveness has been linked to catechol-O-methyltransferase (24) or serotonin transporter (11) polymorphisms in a few recent reports, the magnitude of placebo responses may vary considerably, even within individuals across time and contexts (25). Second, it is likely that placebo responders also respond well to active therapy; therefore, excluding this population of patients would eliminate subjects most prone to benefit from treatment. Antidepressant responses to placebo and active treatment are strongly correlated (26), and in PD, dopamine release in response to levodopa is highly predictive of the magnitude of placeboinduced dopamine release (27). Though it may be premature to use this specific network to exclude participants from trials, the general approach might prove to be beneficial in RCTs.

\section{Acknowledgments}

A.J. Stoessl's work is supported by the Canadian Institutes of Health Research, the Michael J. Fox Foundation, the National Parkinson Foundation, the Pacific Alzheimer Research Foundation, the Pacific Parkinson's Research Institute, and the Canada Research Chairs program.

Address correspondence to: A. Jon Stoessl, Pacific Parkinson's Research Centre, University of British Columbia, Vancouver, British Columbia, Canada V6T 2B5. Phone: 604.822.7935; E-mail: jstoessl@ mail.ubc.ca.

1. Petrovic P, Kalso E, Petersson KM, Andersson J, Fransson $\mathrm{P}$, Ingvar M. A prefrontal nonopioid mechanism in placebo analgesia. Pain. 2010;150(1):59-65.

2. Mayberg HS, et al. The functional neuroanatomy of the placebo effect. Am JPsychiatry. 2002;159(5):728-737.

3. Wager TD, et al. Placebo-induced changes in FMRI in the anticipation and experience of pain.
Science. 2004;303(5661):1162-1167.

4. Goetz CG, et al. Placebo response in Parkinson's disease: comparisons among 11 trials covering medical and surgical interventions. Mov Disord. 2008;23(5):690-699.

5. de la Fuente-Fernández R, Ruth TJ, Sossi V, Schulzer M, Calne DB, Stoessl AJ. Expectation and dopamine release: mechanism of the placebo effect in Parkinson's disease. Science. 2001;293(5532):1164-1166.

6. Strafella AP, Ko JH, Monchi O. Therapeutic application of transcranial magnetic stimulation in Parkinson's disease: the contribution of expectation. Neuroimage. 2006;31(4):1666-1672.

7. Benedetti F, Lanotte M, Colloca L, Ducati A, Zibetti M, Lopiano L. Electrophysiological properties of thalamic, subthalamic and nigral neurons during the anti-parkinsonian placebo response. J Physiol. 2009;587(pt 15):3869-3883.

8. Ko JH, et al. Network modulation following sham surgery in Parkinson's disease. J Clin Invest. 2014;124(8):3656-3666

9. LeWitt PA, et al. AAV2-GAD gene therapy for advanced Parkinson's disease: a double-blind, sham-surgery controlled, randomised trial. Lancet Neurol. 2011;10(4):309-319.

10. Petrovic P, Dietrich T, Fransson P, Andersson J, Carlsson K, Ingvar M. Placebo in emotional processing - induced expectations of anxiety relief activate a generalized modulatory network. Neuron. 2005;46(6):957-969.

11. Furmark T, et al. A link between serotoninrelated gene polymorphisms, amygdala activity, and placebo-induced relief from social anxiety. J Neurosci. 2008;28(49):13066-13074.

12. Amanzio M, Benedetti F, Porro CA, Palermo $\mathrm{S}$, Cauda F. Activation likelihood estimation meta-analysis of brain correlates of placebo analgesia in human experimental pain. Hum Brain Mapp. 2013;34(3):738-752.

13. Schultz W. Behavioral theories and the neurophysiology of reward. Annu Rev Psychol. 2006;57:87-115

14. Haber SN, Kim KS, Mailly P, Calzavara R. Reward-related cortical inputs define a large striatal region in primates that interface with associative cortical connections, providing a substrate for incentive-based learning. J Neurosci. 2006;26(32):8368-8376

15. Stoodley CJ, Schmahmann JD. Functional topography in the human cerebellum: a meta-analysis of neuroimaging studies. Neuroimage. 2009;44(2):489-501.

16. Petrovic P, Kalso E, Petersson KM, Ingvar M. Placebo and opioid analgesia - imaging a shared neuronal network. Science. 2002;295(5560):1737-1740.

17. Kong J, et al. Brain activity associated with expectancy-enhanced placebo analgesia as measured by functional magnetic resonance imaging. J Neurosci. 2006;26(2):381-388.

18. Giacobbe P, Mayberg HS, Lozano AM. Treatment resistant depression as a failure of brain homeostatic mechanisms: implications for deep brain stimulation. Exp Neurol. 2009;219(1):44-52.

19. Wu T, Hallett M. The cerebellum in Parkinson's disease. Brain. 2013;136(pt 3):696-709.

20. Feigin A, et al. Modulation of metabolic brain 
networks after subthalamic gene therapy for Parkinson's disease. Proc Natl Acad Sci U S A. 2007;104(49):19559-19564.

21. Ma Y, Tang C, Spetsieris PG, Dhawan V, Eidelberg D. Abnormal metabolic network activity in Parkinson's disease: test-retest reproducibility. JCereb Blood Flow Metab. 2007;27(3):597-605. 22. Eidelberg D. Metabolic brain networks in neurodegenerative disorders: a functional imaging approach. Trends Neurosci. 2009;32(10):548-557.

23. Kaplitt MG, et al. Safety and tolerability of gene therapy with an adeno-associated virus (AAV) borne GAD gene for Parkinson's disease: an open label, phase I trial. Lancet. 2007;369(9579):2097-2105.

24. Hall KT, et al. Catechol-O-methyltransferase val158met polymorphism predicts placebo effect in irritable bowel syndrome. PLoS One. 2012;7(10):e48135

25. Kaptchuk TJ, et al. Do "placebo responders" exist? Contemp Clin Trials. 2008;29(4):587-595.

26. Kirsch I, Sapirstein G. Listening to Prozac but hearing placebo: A meta-analysis of antidepressant medication. Prev Treat. 1998;1(2):2a.

27. Lidstone SC, et al. Effects of expectation on placebo-induced dopamine release in Parkinson disease. Arch Gen Psychiatry. 2010;67(8):857-865. 